Ireland represents a positive paradigm shift in training toward a return to the psychotherapeutic, rather than solely prescribing, role of the psychiatrist.

1 Martean L, Evans C. Prescribing for personality disorder: qualitative study of interviews with general and forensic consultant psychiatrists. Psychiatr Bull 2014; 38: 116-21.

2 Townsend E, Houston K, Altman DG, Arensman E, Gunnell D, Hazell P, et al. The efficacy of problem-solving treatments after deliberate selfharm: meta-analysis of randomized controlled trials with respect to depression, hopelessness and improvement in problems. Psychol Med 2001: 31: 979-88.

3 Huband N, McMurran M, Evans C, Duggan C. Social problem-solving plus psychoeducation for adults with personality disorder: pragmatic randomised controlled trial. Br J Psychiatry 2007; 190: 307-13.

Melissa Gill is senior registrar in general adult psychiatry at Cavan Mental Health Service, Cavan, Ireland, email: melissa.gill@hse.ie, and MacDara McCauley is consultant general adult psychiatrist at St Brigid's Hospital, Ardee, Co Louth, Ireland.

doi: $10.1192 / \mathrm{pb} .38 .5 .250 \mathrm{a}$

\section{The capacity to attain subjectivity and emotion: the value of continuity of care}

In a refreshing and thought-provoking editorial, Yakeley et al ${ }^{1}$ remind us about the concept of subjectivity. 'Affective subjectivity' is defined as 'the awareness of and reflection on our emotional responses and their influence on our work, and the development of a capacity for self-reflection and emotional attunement with our patients'. ${ }^{1}$ The authors list a number of factors that have led to a reduction in the capacity of psychiatrists to develop and use affective subjectivity. One of these is the loss of continuity of care.

Over the past 10 years we have seen a gradual erosion of the capacity to offer continuity of care to our patients. Psychiatric teams are now fragmented, specialist and largely separate. This enhances splitting within and between teams and makes it difficult for patients to be held in mind for very long.

When I trained as a house officer and junior psychiatrist in the early 1990s, continuity of care was awarded so much importance across the medical specialties that we worked extremely long hours to offer this. An in-depth knowledge of your patient was expected and great emphasis was placed on personally following up their progress and seeing it through. While the long hours were far from ideal, the pendulum has swung so far back that continuity of care is now largely gone. All too often the concept of holistic care is being replaced by diagnoses and treatment plans, rather than formulations based on the biopsychosocial model, ${ }^{2}$ which include affective subjectivity. If a patient is only seen once or twice it is often impossible to expand on developmental and attachment aspects in the history or think about their meaning for the patient, psychiatrist and mental health team.

Without the capacity for doctors to follow patients through, it is very hard to allow subjective feelings to emerge or to use them in the understanding of psychological trauma. I run a Balint-style case discussion group for core trainees in psychiatry. Over the course of the year the barriers to allowing subjective feelings, so well described by Yakeley et al, gradually reduce. For most trainees a deeper understanding of countertransference and how this can help us create an empathic approach to our patients begins to develop. It is frustrating, however, that the majority of the cases brought are not seen again by the trainee. This denies the patient the opportunity to develop a trusting relationship or to experience any kind of attachment (the concept of psychiatric staff as attachment figures is described by Gwen Adshead ${ }^{3}$ ). It also denies the trainees the opportunity to use the understanding gained from the case discussion group to help their patient.

If we are to apply subjectivity and emotion in our work, I think continuity of care needs to be revived. This in turn would enable psychiatrists, once again, to enjoy getting to know patients across a period of time using both subjective and objective skills and thus enhance job satisfaction.

1 Yakeley J, Hale R, Johnston J, Kirtchuk G, Shoenberg P. Psychiatry, subjectivity and emotion - deepening the medical model. Psychiatr Bull 2014; 38: 97-101.

2 Engel G. The need for a new medical model: a challenge for biomedicine. Science 1977; 196: 129-36.

3 Adshead G. Psychiatric staff as attachment figures: understanding management problems in psychiatric services in the light of attachment theory. Br J Psychiatry 1998; 172: 64-9.

Rhona J. Sargeant, consultant medical psychotherapist, Nottingham Psychotherapy Unit, Nottinghamshire Healthcare NHS Trust, Nottingham, UK, email: rhona.sargeant@nottshc.nhs.uk

doi: $10.1192 / \mathrm{pb} .38 .5 .251$

\section{Challenges and opportunities in (neuro)psychiatry}

It would appear that British psychiatry is retreating to a neurophobic position. ${ }^{1}$ The disconnect between psychiatry and its medical foundations is further exacerbated by the lack of medical experience in specialties relevant to psychiatry such as neurology, endocrinology and geriatric medicine. This is related to the constraints placed on training by service provision and the separation of psychiatric and medical services. The Future of Mental Health Services Report, ${ }^{2}$ headed by Prof. Dinesh Bhugra in collaboration with the Mental Health Foundation, called for greater collaboration between psychiatric and general medical services.

Elucidating the nature of mental illness and developing effective treatments requires enthusiastic and talented academics and clinicians. Instead of ideological turf wars, collaboration between disciplines is required to appreciate the nuanced interactions between genetics, biochemistry and the environment.

For example, the classical distinction between affective and psychotic experiences is becoming increasingly blurred. The formation of delusions is associated with a jumping to conclusions' cognitive bias. ${ }^{3}$ This can result in an intolerance of uncertainty and anomalous interpretations of internal or external stimuli. There is renewed focus on the ways in which affective processes can contribute to the formation of delusions and how these mechanisms can be modified using cognitive-behavioural techniques. ${ }^{4}$ These insights from cognitive neuropsychology are substantiated by neuroimaging studies. The salience network, an intrinsic large-scale cerebral network, shows strong connectivity between the anterior cingulate gyrus and insular cortex. This network enables switching between different dynamic brain states. Dysfunction in this network has been implicated in the formation of the key symptoms of psychosis: inappropriate salience attached to ambiguous stimuli can predispose to and perpetuate unusual 
beliefs or delusions. ${ }^{5}$ There is hope that research such as this will begin to have an impact on clinical practice by highlighting these brain-mind links.

As a specialty we need to be far more proactive in promoting psychiatry as clinical neuroscience at both the undergraduate and postgraduate level. We must make sure that the scientific underpinnings of psychiatry are explicit within mental health services and in our interactions with patients and the public in general.

1 Bullmore E, Fletcher P, Jones PB. Why psychiatry can't afford to be neurophobic. Br J Psychiatry 2009; 194: 293-5.

2 Mental Health Foundation. Starting Today: The Future of Mental Health Services, Final Inquiry Report. Mental Health Foundation, 2013.

3 Broome MR, Johns LC, Valli I, Woolley JB, Tabraham P, Brett C, et al Delusion formation and reasoning biases in those at clinical high risk for psychosis. Br J Psychiatry 2007; 191 (suppl 51): s38-42.

4 Garety PA, Freeman, D. The past and future of delusions research: from the inexplicable to the treatable. Br J Psychiatry 2013; 203: 327-33.

5 Palaniyappan L, Liddle PF. Does the salience network play a cardinal role in psychosis? An emerging hypothesis of insular dysfunction. J Psychiatry Neurosci 2012; 37: 17-27.

Ketan D. Jethwa, former academic clinical fellow in general psychiatry, University of Warwick, and CT1 medicine (clinical haematology), Nottingham University Hospitals, Nottingham, UK, email:

ketan.jethwa@nuh.nhs.uk

doi: $10.1192 / p b .38 .5 .251$ a

\section{Reflections on the management of medically unexplained symptoms}

We read with interest the article by Röhricht \& Elanjithara. ${ }^{1}$ They have succinctly presented outcomes associated with delivering a liaison service for medically unexplained symptoms in a community setting. They usefully highlight the absence of current guidelines for the management of medically unexplained symptoms in primary care.

Evidence for treating medically unexplained symptoms has, until now, been dominated by talking therapies. ${ }^{2}$ Patients often describe a perceived mismatch between their physical problems and the offered psychological solutions. They have come to associate body-based problems with body-based solutions and this mismatch may contribute to reluctance in considering psychological therapy. Only $29 \%$ of patients referred to body-oriented psychological therapy (BOPT) participated in assessment and treatment, predominantly from an Asian background. ${ }^{1}$ As Röhricht \& Elanjithara propose, talking therapies may be less acceptable, especially to Black and minority ethnic populations seeking body-based solutions. While the authors have given us an introduction to BOPT, one still does not grasp how this therapy was delivered in practice.
For instance, 106 out of 113 patients received a mental health diagnosis. One wonders what the remainder were thought to have. The importance of this is that most existing models for treatment of medically unexplained symptoms have been limited by the 'uni-professional' nature of treating teams, ${ }^{3}$ including the one described. Distress associated with unmet social needs may indeed undergo 'conversion' to physical symptoms and where expertise is limited to any one professional discipline then outcomes may be affected. This study highlights what may be flawed about the current policy focus on only psychological treatments for these patients. It shows the value of establishing or clarifying diagnoses. It may also be that establishment on psychotropics can help patients to then engage in psychological therapy.

The authors noted that about a quarter of those who received a referral did not attend their initial appointment. Current models of treatment depend on patients turning up for appointments that they may not even remember. They may have been too disabled by their symptoms at the time of appointment or may have considered non-acute services as not useful. These problems are further compounded by the frequently different location of liaison services. Perhaps commissioning for co-location of services and the adoption of assertive outreach approaches may be ways around this block.

Persons with medically unexplained symptoms are often not perceived as having chronic, enduring, mental and physical illness. There is a need for greater awareness of the suffering experienced by this group of patients and the enormous toll that they may take on acute and community services. ${ }^{4}$

1 Röhricht F, Elanjithara T. Management of medically unexplained symptoms: outcomes of a specialist liaison clinic. Psychiatr Bull 2014; doi: 10.1192/pb.bp.112.040733 (published ahead of print 20 February)

2 Creed F, Kronke K, Hennningsen P, Gudi A, White P. Evidence-based treatment. In Medically Unexplained Symptoms, Somatisation and Bodily Distress. Cambridge (eds F Creed, P Henningsen, P Fink): 69-96. Cambridge University Press, 2011.

3 British Pain Society. British Pain Society Response to the Department of Health Improving Access to Psychological Therapies (IAPT) Documents: Long-term conditions positive practice guide, Medically unexplained symptoms positive practice guide. Pain News 2009; Autumn: 14-15.

4 Trærup Andersen NL, Falgaard Eplov L, Trærup Andersen J, Rygaard Hjorthøj C, Birket-Smith M. Health care use by patients with somatoform disorders: a register-based follow-up study. Psychosom 2013; 54: 132-41.

Chizoba Unigwe, specialty doctor, email: chizobaunigwe@nhs.net; Matthew Rowett consultant liaison psychiatrist and Itoro Udo, consultant liaison psychiatrist, Roseberry Park Hopital, Tees, Esk and Wear Valleys NHS Foundation Trust.

doi: $10.1192 / p b .38 .5 .252$

\section{Corrections}

Book review: Play. Psychiatr Bull 2014; 38: 95. The subtitle of this book is 'Experiential Methodologies in Developmental and Therapeutic Settings'. The online version of the review has been amended post-publication, in deviation from print and in accordance with this correction.
Perspectives: Dr Aashish Tagore. Psychiatr Bull 2014; 38: 185-88. In the paper the author is referring to 'classical stages of the grieving process, as described by Prochaska and DiClemente'. This should state 'as described by Kubler-Ross'. doi: $10.1192 /$ pb.38.5.252a 